\title{
Low Temperature Elastic Properties of CuZrTi Bulk Metallic Glass
}

\author{
R. Tarumi ${ }^{1, *}$, M. Hirao ${ }^{1}$, T. Ichitsubo ${ }^{2}$, E. Matsubara ${ }^{2}$, J. Saida ${ }^{3}$ and H. Kato ${ }^{4}$ \\ ${ }^{1}$ Graduate School of Engineering Science, Osaka University, Osaka 560-8531, Japan \\ ${ }^{2}$ Department of Materials Science and Engineering, Kyoto University, Kyoto 606-8501, Japan \\ ${ }^{3}$ Center for Interdisciplinary Research, Tohoku University, Sendai 980-8578, Japan \\ ${ }^{4}$ Institute for Materials Research, Tohoku University, Sendai, 980-8577, Japan
}

Temperature dependent elastic constants $C_{i j}(T)$ of $\mathrm{Cu}_{60} \mathrm{Zr}_{30} \mathrm{Ti}_{10}$ bulk metallic glass (BMG) have been studied using $\mathrm{MHz}$ frequency range electromagnetic acoustic resonance (EMAR) from 4.5 to $300 \mathrm{~K}$. At ambient temperature, the BMG shows low shear/bulk modulus ratio (or equivalently high Poisson ratio) compared with that of corresponding crystalline state dues to the dense random packed glassy structure. Analysis of $C_{i j}(T)$ based on the Einstein type lattice vibration model revealed the notable softening in transverse mode acoustic phonons. This unusual vibrational property suggests the existence of liquid like weakly bonded region in the BMG. [doi:10.2320/matertrans.MJ200764]

(Received November 30, 2006; Accepted May 21, 2007; Published June 25, 2007)

Keywords: elastic constants, CuZrTi bulk metallic glass, ultrasound spectroscopy, acoustic phonon

\section{Introduction}

Because of the loss of long range order in their atomic configuration, bulk metallic glasses (BMGs) are known to have isotropic structural, mechanical and elastic properties. Here, elastic constants and their temperature derivative are related to the configuration and binding nature of atoms in a solid medium, detailed measurement of its temperature dependence $C_{i j}(T)$ therefore illuminates the fundamental vibrational properties and lattice dynamics of the new class of material from the acoustic point of view. ${ }^{1)}$ In addition, a recent study suggested a possibility of correlation between Poisson ratio $v$ and kinetic fragility $m$ defined as the apparent activation energy for shear viscosity of liquid at glass transition temperature $T_{g}{ }^{2}$ ) Since the kinetic fragility distinguishes the glass former liquids from strong to fragile ones it is worthwhile to confirm that such a correlation would work for BMGs or not. For this purpose, precise elastic constants measurements are essential.

Up to now, many experimental works have been reported concerning on the elastic properties of BMGs. ${ }^{3-8)}$ These study revealed remarkable decrease in the shear modulus over bulk modulus ratio $C_{44} / B$ compared with that of corresponding crystalline states. On the other hand, theoretical studies for amorphous solids based on computer simulations and lattice dynamics calculations represented the notable decrease in the shear modulus. ${ }^{9-12)}$ Thus, experimental and theoretical studies show qualitative concurrence, namely metallic glasses have a low shear modulus compared with the corresponding crystalline state. However, explanation of this origin is rather controversial and we are still away from the general consensus. In the present study, the low temperature elastic constant of the $\mathrm{Cu}_{60} \mathrm{Zr}_{30} \mathrm{Ti}_{10}$ BMG has been measured using the ultrasound spectroscopy technique. From the $C_{i j}(T)$ behavior, acoustic Debye temperature, zone-center-mode mean Grüneisen parameter $\gamma$ and Einstein temperature $\Theta_{\mathrm{E}}$ for the respective phonon modes have been derived and interpreted on the basis of the inhomogeneous microstructure model. ${ }^{13)}$

*Corresponding author, E-mail: tarumi@me.es.osaka-u.ac.jp

\section{Experimental Procedure}

\subsection{Material}

The material used in this study is $\mathrm{Cu}_{60} \mathrm{Zr}_{30} \mathrm{Ti}_{10}$ bulk metallic glass prepared by injection molding method. The BMG have a cylinder shape with a diameter of $3.01 \mathrm{~mm}$. Glass transition temperature $T_{g}$ and crystallization temperature $T_{x}$ are determined by differential scanning calorimetry to be 710 and $755 \mathrm{~K}$ with a heating rate of $40 \mathrm{~K} / \mathrm{min}$. Mass densities are determined by the Archimedean method to be $7416 \mathrm{~kg} / \mathrm{m}^{3}$.

\subsection{Ultrasound Spectroscopy}

For elastic constants measurements, we employed the electromagnetic acoustic resonance (EMAR) technique. ${ }^{14)} \mathrm{A}$ specimen is inserted into a solenoid coil (EMAT) and we mount them into a cryogenic chamber. A static magnetic field $(0.2 \mathrm{~T})$ is applied outside from the chamber by pairs of permanent magnets. The solenoid coil was driven by $80 \mu \mathrm{s}$ radio frequency (RF) burst current to induce eddy current on a surface of a specimen. The coupling of eddy current and static magnetic field generates RF Lorentz force which excites ultrasound vibration to the specimen. The detecting process is relies upon the reverse Lorentz force mechanism. In the EMAR method, no coupling agent between the specimen and the transducer is required so that the specimen is acoustically isolated. Thus, the dissipation of vibration energy occurs only inside the specimen which enables us to determine the accurate internal friction $Q^{-1}$. Internal frictions $Q^{-1}$ for respective vibration modes were determined by fitting the free decay curve (ring-down curve) to $\exp (-\alpha t)$ with $Q^{-1}=\alpha / \pi f$, where $\alpha$ and $f$ are attenuation coefficient and resonance frequency, respectively. More details of ultrasound excitation and detection by EMAR are described in a monograph. ${ }^{14)}$ The specimen chamber is filled by He gas about $1.2 \mathrm{~atm}$. and connected with a cold head by a $\mathrm{Cu}$ plate. Temperature of the specimen can be controlled from $4 \mathrm{~K}$ to room temperature. Using the cylindrical specimen, resonance frequencies and internal were measured from room temperature down to approximately $4.5 \mathrm{~K}$.

As shown by Dermarest, stationary point of Lagrangian gives resonance frequencies of an elastic medium, ${ }^{15,16)}$ 


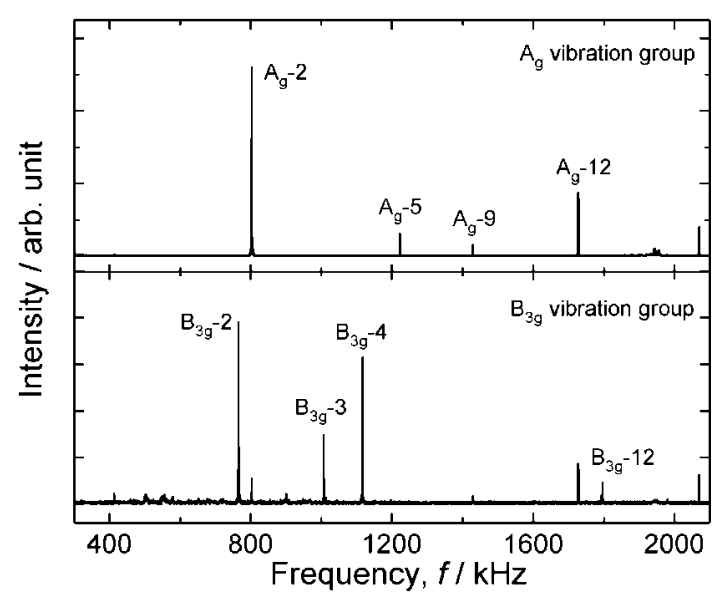

Fig. $1 A_{g}$ and $B_{3 g}$ group free vibration EMAR resonance spectra for the CuZrTi BMG obtained at an ambient temperature. Respective sharp peaks represent resonance frequencies of the specimen indicating that the BMG has low internal friction in the frequency range. The indices in the figure show vibration modes which have been exactly identified by measuring displacement distributions for respective resonance state by a laserDoppler interferometer.

$$
\delta \int \frac{1}{2}\left(C_{i j k l} \varepsilon_{i j} \varepsilon_{k l}-\rho \omega^{2} u_{i} u_{i}\right) d V=0,
$$

Here, $e_{i j}, u_{i}, \rho$ and $\omega$ represent strain, displacement, density and resonance frequency, respectively. Note that summation convention is held for repeated indices in Eq. (1). This variation can be calculated through the Rayleigh-Ritz method by approximating the displacements using Legendre polynominal function. A complete set of elastic constants are then obtained by minimizing the resonance frequency difference between the experimental and calculated ones. Metallic glasses are known to have the following isotropic type elastic constants $C_{i j k l}$ due to the loss of long-range ordering on their atomic configuration,

$$
C_{i j k l}=\lambda \delta_{i j} \delta_{i j}+\mu \delta_{i k} \delta_{j l}+\mu \delta_{i l} \delta_{j k},
$$

where, $\lambda$ and $\mu$ are Lame's constants and $\delta_{i j}$ is the Kronecker delta; $\delta_{i j}=1$ for $i=j$ and 0 for otherwise. Using $\lambda$ and $\mu$, bulk modulus, $B$, Poisson's ratio, $v$, and Young's modulus, $E$, can be expressed as follows; $B=\lambda+2 \mu / 3, v=\lambda /(2 \lambda+$ $2 \mu), E=2 \lambda(1+v)$. In the case of isotropic medium, nonzero components of $C_{i j k l}$ are $C_{1111}, C_{1122}$ and $C_{2323}$ in general. Hereafter, we express them in Voigt notation as $C_{11}, C_{12}$ and $C_{44}$. Note that the independent components are only two since $C_{12}=C_{11}-2 C_{44}$.

\section{Results and Discussion}

\subsection{Room temperature elastic constants}

Prior to low temperature measurements, ambient temperature EMAR measurements have been carried out. Figure 1 shows $A_{g}$ and $B_{3 g}$ vibration group EMAR resonance spectra obtained at $298 \mathrm{~K}$. In the figure, sharp peaks represent the free vibration resonance frequencies of the specimen. Here, vibration modes are exactly identified by measuring the displacement distribution using a laser Doppler interferometer. ${ }^{17)}$ Respective resonance peaks are fitted by the Gaussian function and resonance frequencies are determined from the central frequencies of the fitting curves. Using 9 resonance frequencies shown in Fig. $1\left(\mathrm{~A}_{\mathrm{g}}-2, \mathrm{~A}_{\mathrm{g}}-5, \mathrm{~A}_{\mathrm{g}}-9, \mathrm{~A}_{\mathrm{g}}-12, \mathrm{~B}_{3 \mathrm{~g}}-2\right.$, $\mathrm{B}_{3 \mathrm{~g}}-3, \mathrm{~B}_{3 \mathrm{~g}}-4$ and $\mathrm{B}_{3 \mathrm{~g}}-12$ ), two independent elastic constants $C_{11}$ and $C_{44}$ are determined.

Table 1 summarizes the elastic constants of the BMG obtained at ambient temperature. For comparison, we included the elastic constants of fully crystallized sample which was obtained by a heat treatment over the crystallization temperature. $\Theta_{D}$ in the table shows acoustic Debye temperature defined by the following equation,

$$
\Theta_{\mathrm{D}}=\frac{h}{k_{\mathrm{B}}}\left(\frac{3 N}{4 \pi}\right)^{1 / 3}\left(\frac{\rho}{\mu}\right)^{1 / 3} v_{m},
$$

with

$$
\frac{3}{v_{m}^{3}}=\frac{2}{v_{S}^{3}}+\frac{1}{v_{L}^{3}},
$$

where $k_{\mathrm{B}}$ and $N$ have the usual meanings of statistical mechanics. $h$ is the Planck constant and $\mu$ is the average atomic mass in the system. $v_{S}$ and $v_{L}$ are shear and longitudinal sound wave velocities obtained by solving the Christoffel equation. $v_{m}$ is called mean sound velocity. Diff. in the table represents the elastic constants difference between crystalline and glassy states obtained at an ambient temperature. As seen in the Table, shear modulus $C_{44}$ and shear/bulk modulus ratio $C_{44} / B$ show significant decrease in the glassy state by approximately $30 \%$. According to the theory of elasticity, Poisson ratio $v$ can be expressed in the following form,

$$
v=\frac{1}{2} \frac{3-2 C_{44} / B}{3+C_{44} / B} .
$$

Thus, Poisson ratio monotonically increases with decreasing in $C_{44} / B$ ratio.

\subsection{Low temperature elastic constants and internal friction}

Figure 2 shows a temperature dependence of resonance frequencies obtained by the EMAR measurements. As seen in the figure, all resonance frequencies show monotonic increase with decrease in temperature and unusual properties have not been observed throughout the temperature range ( 4.5 to $298 \mathrm{~K}$ ). Since elastic constants are proportional to the second power of resonance frequency and since elastic constants of usual solids increase with decreasing temperature, the temperature dependence of resonance frequencies found in Fig. 2 would be valid. The temperature dependence of the elastic constants calculated from Fig. 2 is summarized in Fig. 3 and Table 1. In Fig. 3, open circles show the experimental results. Solid lines in the figure show least square fitting result by the following Varshni's function, ${ }^{18)}$

$$
C_{i j}(T)=C_{i j}^{0}-\frac{s}{\exp \left(\Theta_{\mathrm{E}} / T\right)-1} .
$$

In the equation, $C_{i j}^{0}$ and $s$ indicate zero-temperature elastic constants and a fitting parameter including lattice anharmonicity. $\Theta_{\mathrm{E}}$ represents the Einstein temperature for respective phonon modes. From the analysis, $\Theta_{\mathrm{E}}$ are estimated to be 183.0, 122.9 and $265.5 \mathrm{~K}$ for $C_{11}, C_{44}$ and $B$. The $\Theta_{\mathrm{E}}$ represents the temperature in which elastic softening take 
Table 1 Elastic constants $C_{i j}(\mathrm{GPa})$, bulk modulus $B(\mathrm{GPa})$, Poisson's ratio $v$, and acoustic Debye temperature $\Theta_{\mathrm{D}}(\mathrm{K})$ of BMG $(g$ $\mathrm{CuZrTi})$ and corresponding crystalline state $(c-\mathrm{CuZrTi})$.

\begin{tabular}{|c|c|c|c|c|c|c|c|c|}
\hline Material & $T(\mathrm{~K})$ & $C_{11}$ & $C_{12}$ & $C_{44}$ & $B$ & $v$ & $C_{44} / B$ & $\Theta_{\mathrm{D}}(\mathrm{K})$ \\
\hline$g$-CuZrTi & 4.5 & 179.0 & 103.3 & 37.80 & 128.5 & 0.366 & 0.294 & 302.3 \\
\hline$g-\mathrm{CuZrTi}$ & 298 & 169.7 & 102.1 & 33.80 & 124.6 & 0.376 & 0.271 & (285.7) \\
\hline Diff. (\%) & & -11.0 & 6.9 & -29.0 & -2.1 & 12.6 & -27.5 & -15.1 \\
\hline
\end{tabular}

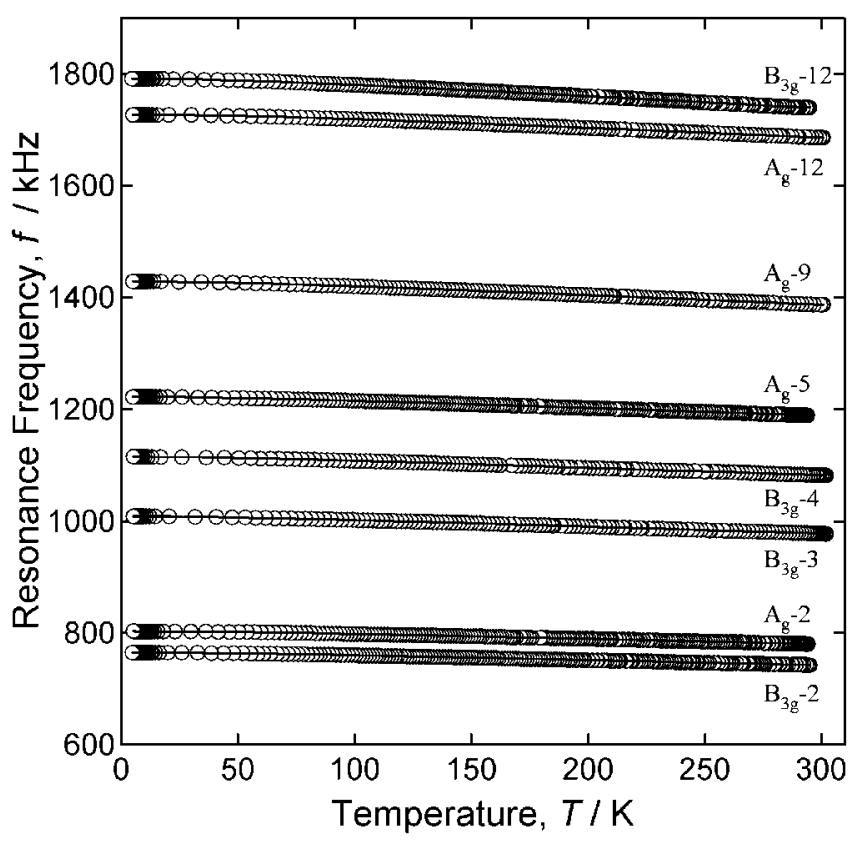

Fig. 2 Temperature dependence of resonance frequencies obtained by the EMAR measurements. Resonance frequencies show monotonic increase with decreasing in temperature and no unusual behavior has been confirmed.

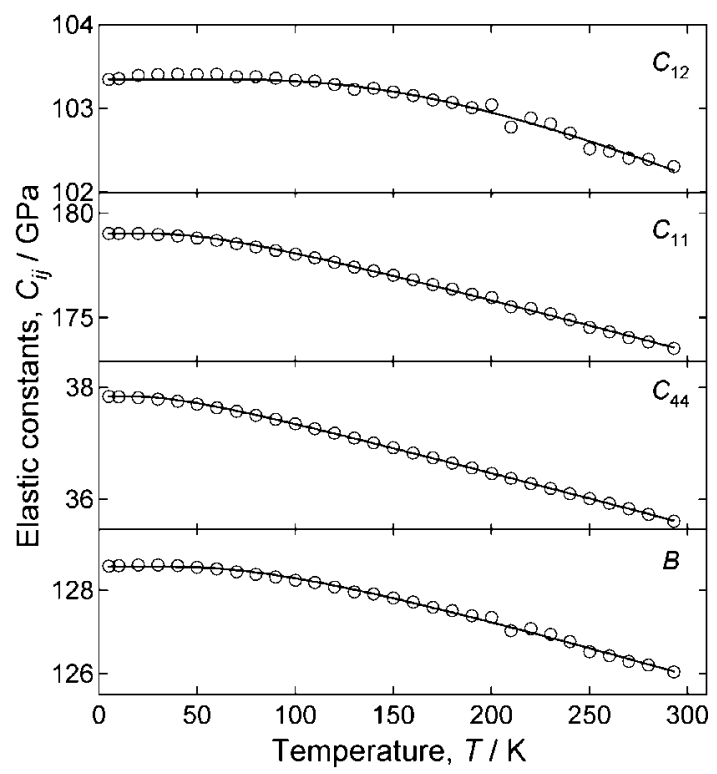

Fig. 3 Temperature dependence of elastic constants obtained from Fig. 2. The solid curves in the figure show the least square fitting results by Varshni's function (Eq. 6).

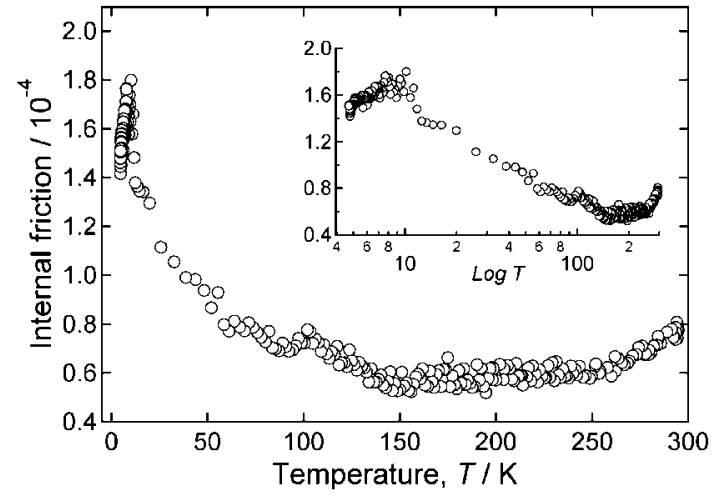

Fig. 4 Temperature dependence of internal friction obtained by ring-down curve measurements. Unusual peak around $10 \mathrm{~K}$ can be clearly confirmed.

place $\left(=\Theta_{E} / 4.5\right)$. In other words, quantum correction is required below $\Theta_{\mathrm{E}} / 4.5$ for respective $C_{i j}$. Thus, the low $\Theta_{\mathrm{E}}$ found in $C_{44}$ suggests the softening in transverse mode acoustic phonon in the BMG. This indicates the existence of liquid-like weakly bonded region in the BMG as suggested by Ichitsubo et al. ${ }^{13)}$

From the temperature dependence of bulk modulus $B$, zone-center-mode mean Grüneisen parameter $\gamma$ has been derived using a following relation, ${ }^{19)}$

$$
d B / d T=3 k_{B} \gamma(\gamma+1) / V_{a}
$$

where $V_{a}$ represents the mean atomic volume. From the analysis, $\gamma$ becomes 1.76 for the BMG. This value is slightly smaller than that of crystalline $\mathrm{Cu}(2.0)$ while almost usual in metallic systems. ${ }^{20)}$ Originally, Grüneisen parameter is defined from the volume dependence of phonon frequency, ${ }^{1)}$ present result indicates that the BMG has an usual level of anharmonicity as a solid.

Finally, it is worthwhile to mention about low temperature unusual internal friction found in the BMG. Figure 4 shows temperature dependence of internal friction obtained from ring-down curve measurements for $\mathrm{B}_{3 \mathrm{~g}}-2$ mode by EMAR. As seen in the figure, internal friction keeps an almost constant value in high temperature side while it start to increase below $100 \mathrm{~K}$ and shows a weak peak around $10 \mathrm{~K}$. Similar unusual features have also been confirmed in another mode. Koning et al. studied low temperature internal friction for $\mathrm{Cu}_{70} \mathrm{Zr}_{30}$ amorphous alloy and reported essentially similar unusual behavior at low temperature side. ${ }^{21)}$ According to this work, the unusual internal friction behavior would be due to the tunneling of phonon between two level states. Unfortunately, however, present study has not been carried out further analysis for them since our primary interest is to clarify the elastic properties of the BMG. Detailed analysis for the internal friction behavior is of our future work. 


\section{Conclusions}

In summary, the elastic constants of $\mathrm{Cu}_{60} \mathrm{Zr}_{30} \mathrm{Ti}_{10}$ bulk metallic glass has been measured by EMAR technique from ambient temperature down to $4.5 \mathrm{~K}$. The BMG showed notable decrease in the $C_{44} / \mathrm{B}$ ratio (or increase in Poisson ratio) compared with the corresponding crystalline state due to the dense random packed glassy structure. Analysis for $C_{i j}(T)$ based on the Einstein-type lattice vibration model quantitatively suggested the softening in transverse mode acoustic phonon. Based on the inhomogeneous microstructure model, the softening in transverse phonon can be explained by the existence of liquid like weakly bonded region in the dense random packed glassy structure even in a deep glassy state.

\section{REFERENCES}

1) M. Born and K. Huang: "Dynamical Theory of Crystal Lattice" (Claredon Press, Oxford, 2002).

2) V. N. Novikov and A. P. Sokolov: Nature 431 (2004) 961.

3) B. Golding, B. G. Bagley and F. S. Hsu: Phys. Rev. Lett. 29 (1972) 68

4) T. Ichitsubo, E. Matsubara, S. Kai and M. Hirao: Acta Mat. 52 (2004) 423.
5) T. Ichitsubo, S. Kai, H. Ogi, M. Hirao and K. Tanaka: Scr. Mater. 49 (2003) 267.

6) E. F. Lambson, W. A. Lambson, J. E. Macdonald, M. R. J. Gibbs, G. A. Saunders and D. Turnbull: Phys. Rev. B 33 (1986) 2380.

7) W. H. Wang, Z. X. Bao, C. X. Liu, D. Q. Zhao and J. Eckert: Phys. Rev. B 61 (2000) 3166.

8) W. H. Wang, F. Y. Li, M. X. Pan, D. Q. Zhao and R. J. Wang: Acta Mater. 52 (2004) 715.

9) D. Weaire, M. F. Ashby, J. Logan and M. J. Weins: Acta Met. 19 (1971) 779 .

10) F. C. Lackmann: Phys. Rev. B 22 (1980) 2744.

11) K. Maeda and S. Takeuchi: Phil. Mag. A 44 (1981) 643.

12) D. S. Franzblau and J. Tersoff: Phys. Rev. Lett. 68 (1992) 2172.

13) T. Ichitsubo, E. Matsubara, T. Yamamoto, H. S. Chen, N. Nishiyama, J. Saida and K. Anazawa: Phys. Rev. Lett. 95 (2005) 245501.

14) M. Hirao and H. Ogi: "EMATs for Science and Industry" (Kluwer, Boston, 2003)

15) H. H. Dermarest Jr.: J. Acous. Soc. Am. 49 (1971) 768.

16) I. Ohno: J. Phys. Earth 24 (1976) 355.

17) H. Ogi, K. Sato, T. Asada and M. Hirao: J. Acoust. Soc. Am. 112 (2002) 2553.

18) Y. P. Varshni: Phys. Rev. B 2 (1970) 3952.

19) H. Ledbetter: Phys. Status Solidi (b) 181 (1994) 81.

20) M. Levy, H. E. Bass and R. R. Stern: "Handbook of Elastic Properties of Solids, Liquids, and Gases" (Academic Press, San Diego, 2001).

21) R. Konig, M. A. Ramos, I. Usherov-Marshak, J. Arcas-Guijarro, A. Hernando-Maneru and P. Esquinazi: Phys. Rev. B 65 (2002) 180201(R). 